\title{
THE HYPERQUASICENTER OF A FINITE GROUP. I
}

\author{
N. P. MUKHERJEE ${ }^{1}$
}

ABSTRACr. The quasicenter is a generalisation of the idea of the center of a group and this has been used to define the hyperquasicenter. The concept that is essentially involved is that of quasinormality. The quasicenter has been shown to be nilpotent and the hyperquasicenter has been identified as the largest supersolvably immersed subgroup.

Introduction. The concept of a quasicentral element of a group $G$ is generalisation of the idea of an element in the center. An element $g \in G$ is called a quasicentral element (QC-element) of $G$ if $\langle g\rangle\langle x\rangle$ $=\langle x\rangle\langle g\rangle \forall x \in G$. This leads to the definition of the quasicenter $Q(G)$ which is the subgroup generated by all quasicentral elements and it can be easily shown to be a characteristic subgroup. The principal results related to quasicentral elements and the quasicenter are: (1) If $x \in G$ is a $Q C$-element then $x^{r}$ is a $Q C$-element for all integers $r$. (2) $Q(G)$ is nilpotent.

The quasicenter leads to the definition of the hyperquasicenter in the following manner. Let $Q_{0}=1$ and $Q_{i+1} / Q_{i}$ be the quasicenter of $G / Q_{i}$. This gives an ascending chain of characteristic subgroups as follows: $1=Q_{0} \subset Q_{1} \subset Q_{2} \subset \cdots \subset Q_{n}=Q^{*}$. The terminal member $Q^{*}$ of this series (termed upper quasicentral series) is called the hyperquasicenter of $G$. The principal result described in the paper is that the hyperquasicenter has been identified as the largest supersolvably immersed subgroup of $G$.

Definition 1.1. An element $x \in G$ is a quasicentral element (QCelement) iff $\langle x\rangle\langle y\rangle=\langle y\rangle\langle x\rangle$ for all $y$ in $G$.

TheOREM 1.2. If $x$ is a QC-element of a group $G$ then $x^{r}$ is a QC-element for all integers $r$.

Proof. Let $y$ be an arbitrary but fixed element of $G$. Then we must prove $\left\langle x^{r}\right\rangle\langle y\rangle=\langle y\rangle\left\langle x^{r}\right\rangle$ for all integers $r$. We argue by induction on $|G|$. Thus we can assume that $G=\langle x, y\rangle$. Set $X=\langle x\rangle$ and $Y=\langle y\rangle$.

Received by the editors March 13, 1970.

AMS 1969 subject classifications. Primary 2040.

Key words and phrases. Quasicentral element (QC-element), quasicenter, hyperquasicenter.

1 The author wishes to thank the referee for his useful suggestions.

Part of the paper was included in a doctoral thesis written at Michigan State University, 1968. 
Then $G=X Y$. There are two cases. First consider that either $X$ or $Y$ contains a nontrivial normal subgroup $Z$ of $G$. Set $\bar{G}=G / Z, \bar{X}=X Z / Z$ and $\bar{Y}=Y Z / Z$. By induction $\bar{X}^{r} \bar{Y}=\bar{Y} \bar{X}^{r}$. So $X^{r} Y Z=Y X^{r} Z$. By the assumption on $Z, X^{r} Y=Y X^{r}$ and $x^{r}$ is a $Q C$-element in this case. In the remaining case neither $X$ nor $Y$ contains a normal subgroup of $G$. Hence $X \cap Y=1$. Then $|G|=|X| \cdot|Y|$.

Suppose that $|Y| \geqq|X|$. Then as $Y$ is not normal in $G, G$ has a double coset of the form $Y x Y \neq Y x$. Then

$$
Y x Y=|Y|^{2} /\left|Y \cap Y^{x-1}\right| .
$$

Set $Y_{0}=Y \cap Y^{x^{-1}}$. Then $Y_{0}^{x} \subseteq Y$. As $Y$ is cyclic, $Y_{0}^{x}=Y_{0}$. This implies that $Y_{0} \subset G=X Y$. Hence $Y_{0}=1$. Now we have $|G|>|Y x Y|$ $=|Y|^{2} /\left|Y_{0}\right|=|Y|^{2} \geqq|Y| \cdot|X|=G$, a contradiction. The same argument applies if $|X| \geqq|Y|$. Hence the theorem follows.

Definition 1.3. The quasicenter $Q(G)$ of a group $G$ is the subgroup generated by all QC-elements of $G$.

The following example shows that every element of $Q(G)$ need not be a $Q C$-element and also that the product of any two $Q C$-elements is not necessarily a $Q C$-element.

EXAMPLE. Let $G=\langle a, b\rangle\langle x\rangle=\langle x\rangle\langle a, b\rangle$ where $a^{3}=1, b^{3}=1, a b=b a$, $a^{x}=a^{2}, b^{x}=b, x^{2}=1 . Q(G)=\langle a, b\rangle$ and $a, b$ are QC-elements of $G$. But $a b \in Q(G)$ is not a $Q C$-element of $G$.

The structure of $Q(G)$ is investigated next and the following lemma is necessary for that.

LEMma 1.4. Every Sylow subgroup of the quasicenter $Q(G)$ of a group $G$ is generated by $Q C$-elements of $G$.

Proof. Let $\left\{x_{1}, x_{2}, \cdots, x_{t}\right\}=X$ be the set of all QC-elements of $G$ and note that $\langle X\rangle=Q(G)$. Each $x_{i}$ can be written as $x_{i}=y_{i} z_{i}=z_{i} y_{i}$, where $y_{i}$ is a $p$-element, $z_{i}$ is a $p^{\prime}$-element, $i=1,2, \cdots, t$, and $y_{i}, z_{i}$ are $Q C$-elements of $G$ being powers of $x_{i}$. Define $X_{p}=\left\{y_{1}, y_{2}, \cdots, y_{t}\right\}$ and let $\theta$ be any automorphism of $G$. The image of the QC-element $x_{i}$ under $\theta$ is $y_{i}^{\theta} z_{i}^{\theta}$ and it is a QC-element of $G$. Therefore $y_{i}^{\theta} z_{i}^{\theta}$ is in $X$ and $y_{i}^{\theta}$ is in $X_{p}$. Consequently $X_{p}$ is a normal set. Now $y_{i} \forall i$ being a $Q C$ element of $G$ it follows that $\left\langle X_{p}\right\rangle$ is a $p$-group and therefore if $P$ is a Sylow $p$-subgroup of $Q(G)$ then $\left\langle X_{p}\right\rangle \subseteq P$. But $Q(G)=\left\langle x_{1}\right\rangle \cdot\left\langle x_{2}\right\rangle$. $\cdots \cdot\left\langle x_{t}\right\rangle=\left\langle y_{1}\right\rangle \cdot\left\langle y_{2}\right\rangle \cdot \cdots \cdot\left\langle y_{t}\right\rangle \cdot\left\langle z_{1}\right\rangle \cdot\left\langle z_{2}\right\rangle \cdot \cdots \cdot\left\langle z_{t}\right\rangle$. Hence $|P|$ $=\left|\left\langle y_{1}\right\rangle \cdot\left\langle y_{2}\right\rangle \cdots \cdot\left\langle y_{t}\right\rangle\right|$ and so $P=\left\langle X_{p}\right\rangle$. The assertion in the lemma therefore follows.

TheOREM 1.5. The quasicenter of a group $G$ is nilpotent. 
Proof. Let $P_{1}$ and $P_{2}$ be two Sylow subgroups of $Q(G)$, corresponding to the prime divisor $p$ of $Q(G)$. Let $P_{1}=\left\langle g_{1}, g_{2}, \cdots, g_{n}\right\rangle$, where $g_{i}$ is a $Q C$-element of $G$ for $i=1,2, \cdots, n$. If $g_{i} \notin P_{2}$ for some $i$, then consider $P_{2}\left\langle g_{i}\right\rangle=\left\langle g_{i}\right\rangle P_{2}$. It is a $p$-group whose order is larger than $\left|P_{2}\right|$ a contradiction. Hence $P_{1} \subseteq P_{2}$ and so $P_{1}=P_{2}$, since $\left|P_{1}\right|=\left|P_{2}\right|$. Thus there is a unique Sylow subgroup of $Q(G)$ corresponding to each prime divisor of $Q(G)$.

Hence $Q(G)$ is nilpotent. If $H \triangleleft G$ then $Q(H)$ in general neither contains nor is included in $Q(G)$ always. For $G=A_{4}$ and $H \triangleleft G$, $Q(H) \Phi Q(G)$ and if $G=$ the quaternion group, $H \triangleleft G$ then $Q(G)$ $\Phi Q(H)$. This leads to the investigation of the relationship of the quasicenter of a group $G$ and quasicenters of subgroups with additional restrictions besides normality imposed on the subgroups.

THEOREM 1.6. If a group $G$ is the direct product of subgroups $H$ and $K$ then $Q(G) \subseteq Q(H) \times Q(K)$. Further, if $(|H| \cdot|K|)=1$ then $Q(G)$ $=Q(H) \times Q(K)$.

REMARK. If $G=H \times K$ it is not in general true that a $Q C$-element of $H$ or $K$ will be a $Q C$-element of $G$. That is why the equality of $Q(G)$ and $Q(H) \times Q(K)$ in general seems improbable. The following example confirms this assertion.

EXAMPLE. Let $G=\langle a\rangle\langle b\rangle=\langle b\rangle\langle a\rangle$, where $|a|=3,|b|=9, a^{-1} b a=b^{4}$. Note that ' $a$ ' is a QC-element. Now if $S=\left\langle a_{1}\right\rangle \cdot\left\langle b_{1}\right\rangle \times\left\langle a_{2}\right\rangle \cdot\left\langle b_{2}\right\rangle=G_{1} \times G_{2}$ where $G_{1}$ and $G_{2}$ are copies of $G$ then $a_{1}$ is not a QC-element of $S$ since $\left\langle a_{1}\right\rangle$ does not permute with $\left\langle b_{1} b_{2}\right\rangle$.

The definition of the hyperquasicenter follows from the definition of the quasicenter in an exactly identical manner as that of the hypercenter follows from the definition of the center. Let $G$ be a group and suppose $Q_{0}=1$ and $Q_{i+1} / Q_{i}$ is the quasicenter of $G / Q_{i}$. Then the terminal member $Q^{*}$ of the chain $1=Q_{0} \subset Q_{1} \subset Q_{2} \subset \cdots \subset Q_{n}=Q^{*}$ of characteristic subgroups is called the hyperquasicenter of $G$. We shall now prove the main result of the paper. For the sake of completeness we include the following definition.

Definition 1.7. A subgroup $M$ of a group $G$ is called supersolvably immersed in $G$ if for each homomorphism of $G$ the image of $M$ contains a cyclic subgroup which is normal in the homomorphic image of G.

THEOREM 1.8. The hyperquasicenter of a group $G$ is the largest supersolvably immersed subgroup of $G$.

Proof. Let $Q^{*}$ be the hyperquasicenter of $G$. We prove first that 
every supersolvably immersed subgroup of $G$ is contained in $Q^{*}$ and this we do by induction on the order of $G$. For $|G|=1$ this is true and assume it to be true for all groups of order less than $|G|$. If $M$ is a supersolvably immersed subgroup of $G$ then it shall contain a cyclic subgroup $\langle a\rangle$ which is normal in $G$ and note that $M /\langle a\rangle$ is a supersolvably immersed subgroup of $G /\langle a\rangle$. Since $\langle a\rangle$ is a normal subgroup of $G$ it follows that $\langle a\rangle\langle x\rangle=\langle x\rangle\langle a\rangle \forall x \in G$. Therefore ' $a$ ' is a QC-element of $G$ and $\langle a\rangle \subseteq Q^{*}$. Hence by induction $M /\langle a\rangle \subseteq Q^{*} /\langle a\rangle$. (It is not difficult to show that if $T$ is a normal subgroup contained in the hyperquasicenter $Q^{*}$ of a group $G$ then the hyperquasicenter of $G / T$ equals $Q^{*} / T$.) Thus $M \subseteq Q^{*}$.

We now prove that the hyperquasicenter is a supersolvably immersed subgroup of $G$ and the main step in proving this is to show that the quasicenter is supersolvably immersed as in that case supersolvable immersion of the hyperquasicenter will immediately follow.

Let $P$ be a Sylow $p$-subgroup of the quasicenter $Q(G)$ of $G . P$ is generated by $Q C$-elements and let $x$ be a $Q C$-generator of $P$. Suppose $y$ is a p-element of $G$. Set $X=\langle x\rangle$ and $Y=\langle y\rangle$. Then $X Y=Y X$ by assumption and so $X Y$ is a group. Therefore $x^{y}=x^{a} y^{b}$ for some integers $a$ and $b$. But $y^{b}=x^{-a} x^{y}$ is a p-element belonging to $P$. Thus $y^{b}=1$ and so $x^{y}=x^{a} . X$ is therefore normalised by all $p$-elements of $G$. But from the structure of the automorphism group of a cyclic $p$-group it now follows that $X$ is centralised by $y^{p-1}$ where $y$ is any $p$-element of $G$. Since $P$ is generated by $Q C$-elements the above statement implies that $\left[P / D(P), y^{p-1}\right]=1$ for all $p$-elements of $G$ where $D(P)$ is the Frattini subgroup of $P$. From this it follows that $\left[P, y^{p-1}\right]=1$. By Theorem 6.1 of Baer in [6] it follows therefore that $P$ is supersolvably immersed and so the quasicenter $Q(G)$ is also supersolvably immersed. The assertion in the theorem therefore follows.

\section{REFERENCES}

1. R. Baer, The hypercenter of a group. I, Acta Math. 89 (1953), 165-208. MR $15,395$.

2. - Das Hyperzentrum einer Gruppe. II, Arch. Math. 4 (1953), 86-96. MR $15,396$.

3. - Das Hyperzentrum einer Gruppe. III, Math. Z. 59 (1953), 299-338. MR $15,598$.

4. - Das Hyperzentrum einer Gruppe. IV, Arch. Math. 5 (1954), 56-59. MR 15, 776.

5. - Group elements of prime power index, Trans. Amer. Math. Soc. 75 (1953), 20-47. MR 14, 1059.

6. - Principal factors, maximal subgroups and conditional identities of finite groups, Illinois J. Math. 13 (1969), 1-52. MR 38 \#5933. 
7. W. E. Deskins, On quasinormal subgroups of finite groups, Math. Z. 82 (1963), 125-132. MR 27 \#3699.

8. N. Ito and J. Szep, Über die Quasinormalteiler von endlichen Gruppen, Acta Sci. Math. (Szeged) 23 (1962), 168-170. MR 25 \#2119.

9. M. Suzuki, Structure of a group and the structure of its lattice of subgroups, Ergebnisse der Mathematik und ihrer Grenzgebiete, Neue Folge, Heft 10, SpringerVerlag, Berlin, 1956. MR 18, 715.

10. H. Wielandt, Über das Produkt paarweise Vertauschbarer nilpotenter Gruppen, Math. Z. 55 (1951), 1-7. MR 13, 621.

West Virginia University, Morgantown, West Virginia 25606 